\title{
Pinning Control of Coupled Networks with Time-Delay
}

\author{
Kun Yuan ${ }^{1}$, Gang Feng ${ }^{2}$ and Jinde $\mathrm{Cao}^{3, *}$ \\ ${ }^{1}$ Southeast University, School of Automation, China \\ ${ }^{2}$ City University of Hong Kong, Department of Manufacturing Engineering and Engineering Management, Hong Kong \\ ${ }^{3}$ Southeast University, Department of Mathematics, China
}

\begin{abstract}
This paper studies the global synchronization for undirect and direct coupled networks with delay by employing Lyapunov functional method and Kronecker product technique. A novel pinning scheme is proposed and some sufficient conditions are given to ensure the global synchronization of coupled networks. Finally, an example is given to show the effectiveness of the proposed pinning scheme.
\end{abstract}

Keywords: Coupled networks, pinning control, synchronization, delayed neural networks, lyapunov functional.

\section{INTRODUCTION}

Various large-scale and complicated systems can be modelled by complex networks, such as the Internet, genetic networks, ecosystems, electrical power grids, the social networks, and so on. A complex network is a large set of interconnected nodes, which can be described by the graph with the nodes representing individuals in the graph and the edges representing the connections among them. The most remarkable recent advances in study of complex networks are the developments of the small-world network model [1] and scale-free network model [2], which have been shown to be very closer to most real-world networks as compared with the random-graph model $[3,4]$. Thereafter, small-world and scale-free networks have been extensively investigated.

The dynamical behaviors of complex networks have become a focal topic of great interest, particularly the synchronization phenomena, which is observed in natural, social, physical and biological systems, and has been widely applied in a variety of fields, such as secure communication, image processing and harmonic oscillation generation. It is noted that the dynamical behavior of a complex network is determined not only by the dynamical rules governing the isolated nodes, referred to as self-dynamics, but also by information flow along the edges, which depends on the topology of the network. For a given network with identical node dynamics and diffusive coupling, two key factors influencing the network synchronization are the network coupling strength and the coupling configuration matrix. Synchronization in an array of linearly coupled dynamical systems was investigated in [8]. Later, many results on local, global and partial synchronization in various coupled systems have also been obtained in [9-15]. As a special case of coupled systems, coupled neural networks have also been found to exhibit complex behaviors and their synchronization has been investigated by many researchers, e.g. [16-23].

*Address correspondence to this author at the Southeast University Department of Mathematics, China; Tel: +86-25-83792315; Fax: 86-25-83792316; E-mail: jdcao@seu.edu.cn
In the case that the whole network cannot synchronize by itself, some controllers should be designed and applied to force the network to synchronize. A special control strategy called pinning control [24] is designed to achieve synchronization of complex networks by controlling only a fraction of the nodes or even a single node [29] over the whole network, which has become a common technique for control, stabilization and synchronization of coupled dynamical systems [25-32]. In [30], both specific and random pinning schemes were studied, where specific pinning scheme of the nodes with large degrees is shown to require a smaller number of controlled nodes than random pinning scheme. In [26], an adaptive pinning controller was proposed, and it was found that the nodes with very low degrees should be pinned first when the coupling strength is small. However, the design of pinning control in those references was for the undirected network, in other words, the interaction topology is bidirectional, thus the coupling matrix is symmetrical. In practice, the directed network is important and unidirectional communication exists in application. This paper aims to investigate the following issues for undirected and directed networks:(a) What kind of pinning schemes should be chosen for the given undirected and directed network to achieve synchronization? (b) What type of controllers should be designed to ensure the network synchronization? (c) How to choose the coupling strength for a network with a fixed topological structure to achieve synchronization?

The rest of the paper is organized as follows. In section 2, some preliminary definitions and lemmas are briefly outlined. Some synchronization criteria are given and a selective scheme is proposed in Section 3. An illustrative simulation is given to verify the theoretical analysis in Section 4. Conclusions are finally drawn in Section 5.

Notation. Throughout this paper, for real symmetric matrices $X$ and $Y$, the notation $X \geq Y$ (respectively, $X>Y$ ) means that the matrix $X-Y$ is positive semi-definite (respectively, positive definite). The superscript " $T$ " represents the transpose. For a matrix $A,\|A\|$ denotes the 
spectral norm defined by $\|A\|=\left(\lambda_{\max }\left(A^{T} A\right)\right)^{1 / 2}$. Matrix dimensions, if not explicitly stated, are assumed to be compatible for algebraic operations.

\section{MODEL DESCRIPTION AND PRELIMINARIES}

Consider a dynamical network consisting of $N$ identical and diffusively coupled nodes, with each node being an ndimensional delayed neural network. The state equations of the network are

$$
\dot{x}_{i}(t)=-D x_{i}(t)+A f\left(x_{i}(t)\right)+B f\left(x_{i}(t-\tau)\right)+I(t)+c \sum_{j=1, j \neq i}^{N} G_{i j} \Gamma\left(x_{j}(t)-x_{i}(t)\right)
$$

where $x_{i}(t)=\left(x_{i 1}(t), x_{i 2}(t), \cdots, x_{i n}(t)\right)^{T} \in \mathrm{R}^{n}$ is the state vector of the $i$ th node; $D=\operatorname{diag}\left(d_{1}, d_{2}, \cdots, d_{n}\right)>0$ denotes the rate with which the cell $i$ resets its potential to the resting state when isolated from other cells and inputs; $A \in \mathrm{R}^{n \times n}$, and $B \in \mathrm{R}^{n \times n}$ represent the connection weight matrix and the delayed connection weight matrix respectively; $f\left(x_{i}(\cdot)\right)=\left[f_{1}\left(x_{i 1}(\cdot)\right), f_{2}\left(x_{i 2}(\cdot)\right), \cdots, f_{n}\left(x_{i n}(\cdot)\right)\right]^{T} \in \mathrm{R}^{n} \quad$ is $\quad$ activation function; $I(t)$ is the input vector of each node; $c \in \mathrm{R}^{1}$ is the coupling strength; $\Gamma \in \mathrm{R}^{n \times n}$ is the inner coupling matrix; $G=\left(G_{i j}\right)_{N \times N}$ is the coupling configuration matrix representing the topological structure of the network, where $G_{i j}$ is defined as follows: if there exists a connection between node $i$ and node $j, G_{i j}>0$, otherwise $G_{i j}=0 \quad(j \neq i)$, and the diagonal elements of matrix $G$ are defined by

$$
G_{i i}=-\sum_{j=1, j \neq i}^{N} G_{i j} \text {, }
$$

which ensures the diffusion that $\sum_{j=1}^{N} G_{i j}=0$. Equivalently, network (1) can be rewritten in a form as follows:

$$
\dot{x}_{i}(t)=-D x_{i}(t)+A f\left(x_{i}(t)\right)+B f\left(x_{i}(t-\tau)\right)+I(t)+c \sum_{j=1}^{N} G_{i j} \Gamma x_{j}(t), i=1,2, \cdots, N
$$

Suppose that the coupled network (2) is connected in the sense that there are no isolated clusters. Then the coupling matrix $G$ is irreducible.

We define the set

$\mathrm{S}=\left\{x=\left(x_{1}^{T}, x_{2}^{T}, \cdots, x_{N}^{T}\right)^{T} \in \mathrm{R}^{n N}: x_{i}=x_{j}, i, j=1,2, \cdots, N\right\} \quad$ as $\quad$ the synchronization manifold for network (2).

In addition, the following assumption is needed,

(H) $f_{k}(\cdot): \mathrm{R} \rightarrow \mathrm{R}$ is Lipschitz continuous and monotonically nondecreasing, i.e., $\forall x, y \in \mathrm{R}$, there are real positive numbers $l_{k}$ such that

$0 \leq \frac{f_{k}(x)-f_{k}(y)}{x-y} \leq l_{k}, \quad k=1,2, \cdots, n$.

For convenience, we denote $L=\operatorname{diag}\left(l_{1}, l_{2}, \cdots, l_{n}\right)$.

Definition 1. Matrix $G=\left(G_{i j}\right)_{N \times N}$ is said to belong to class $A_{1}$, denoted as $G \in A_{1}$, if

(i) $G_{i j} \geq 0, i \neq j, G_{i i}=-\sum_{j=1, j \neq i}^{N} G_{i j}, i=1,2, \cdots, N$

(ii) $G$ is irreducible.
If $G \in A_{1}$ is symmetrical, then we say that $G$ belongs to class $A_{2}$, denoted as $G \in A_{2}$.

Lemma 1.[5] If matrix $G \in A_{1}$, then $\operatorname{rank}(G)=N-1$, i.e. 0 is an eigenvalue of $G$, and all nonzero eigenvalues of $G$ have a negative real part.

Lemma 2.[6] If matrix $G \in A_{1}$, then

1. $\mathbf{1}=(1,1, \cdots, 1)^{T}$ is the right eigenvector of $G$ corresponding to eigenvalue 0 with multiplicity 1 , i.e., $G \cdot \mathbf{1}=0$;

2. Let $\xi=\left(\xi_{1}, \xi_{2}, \cdots, \xi_{N}\right)^{T}$ (without loss of generality, assume $\left.\sum_{i=1}^{N} \xi_{i}=1\right)$ be the left eigenvector of $G$ corresponding to the eigenvalue 0 , i.e. $\xi^{T} G=0$. Then $\xi_{i}>0$, $i=1,2, \cdots, N$.

Definition 2. For any positive integers $p, q, r, s$, we define the Kronecker product of two matrices $A \in \mathrm{R}^{p \times q}$, $B \in \mathrm{R}^{r \times s}$ as follows

$A \otimes B=\left[\begin{array}{ccc}a_{11} B & \cdots & a_{1 q} B \\ \vdots & \ddots & \vdots \\ a_{p 1} B & \cdots & a_{p q} B\end{array}\right] \in \mathrm{R}^{p r \times q s}$.

Lemma 3. By the definition of Kronecker product, the following properties hold:

1. $(A \otimes B)^{T}=A^{T} \otimes B^{T} ;$

2. $(\alpha A) \otimes B=A \otimes(\alpha B)$, where $\alpha$ is a real number;

3. $(A \otimes B)(C \otimes D)=(A C) \otimes(B D)$.

Lemma 4 [29] If the matrix $R=\left(r_{i j}\right) \in \mathrm{R}^{N \times N}$ is irreducible, $r_{i j}=r_{j i} \geq 0$, for $i \neq j$, and $\sum_{j=1}^{N} r_{i j}=0$ with $\operatorname{rank}(R)=N-1$, for all $i=1,2, \cdots, N$, then all eigenvalues of the matrix

$\left[\begin{array}{cccc}r_{11}-\varepsilon & r_{12} & \cdots & r_{1 N} \\ r_{21} & r_{22} & \cdots & r_{2 N} \\ \vdots & \vdots & \ddots & \vdots \\ r_{N 1} & r_{N 2} & \cdots & r_{N N}\end{array}\right]$

are negative for any positive constant $\varepsilon$.

Lemma 5. [7] Let $\left\{\lambda_{i} \mid i=1,2, \cdots, n\right\}$ and $\left\{\mu_{j} \mid j=1,2, \cdots, m\right\}$ be the eigenvalues of matrices $A$ and $B$, respectively. Then

$\left\{\lambda_{i} \mu_{j} \mid i=1,2, \cdots, n, \quad j=1,2, \cdots, m\right\}$

are the eigenvalues of $A \otimes B$.

\section{MAIN RESULTS}

\subsection{Undirected Networks}

Note that a solution to an isolated node satisfies

$\frac{d s(t)}{d t}=-D s(t)+A f(s(t))+B f(s(t-\tau))+I(t)$

To realize the synchronization of network (2), pinning control will be added to some of its nodes. Here, the pinning strategy is applied to a small fraction of the nodes in network 
(2). Suppose that the nodes $i_{1}, i_{2}, \cdots, i_{l}$ are selected, where $l<N$. Without loss of generality, rearrange the order of the nodes in the network, and let the first $l$ nodes be controlled. Thus the pinning controlled network can be described by

$\dot{x}_{i}(t)=-D x_{i}(t)+A f\left(x_{i}(t)\right)+B f\left(x_{i}(t-\tau)\right)+I(t)$

$+c \sum_{j=1}^{N} G_{i j} \Gamma x_{j}(t)+u_{i}, i=1,2, \cdots, N$,

where $G_{i j}=G_{j i}$, i.e., $G \in A_{2}$,

$u_{i}=-c k_{i} \Gamma\left(x_{i}(t)-s(t)\right)$,

and the feedback gains $k_{i}$ are positive constants for $i=1,2, \cdots, l$ and $k_{i}=0$ for $i=l+1, \cdots, N$.

Our objective is then to investigate the synchronization of network (5) with respect the isolated node $s(t)$. Denote $e_{i}(t)=x_{i}(t)-s(t)$, then the following error dynamical system is obtained:

$\dot{e}_{i}(t)=-D e_{i}(t)+A\left[f\left(x_{i}(t)\right)-f(s(t))\right]$

$+B\left[f\left(x_{i}(t-\tau)\right)-f(s(t-\tau))\right]+c \sum_{j=1}^{N} G_{i j} \Gamma e_{j}(t)-c k_{i} \Gamma e_{i}(t)$,

$i=1,2, \cdots, N$

Theorem 1. Suppose that Assumption $(H)$ holds. The controlled undirected network (5) globally synchronizes to (4) if there is a symmetric matrix $P \in \mathrm{R}^{n \times n}$ and a diagonal nonnegative matrix $K=\operatorname{diag}\left(k_{1}, k_{2}, \cdots, k_{l}, 0,0 \cdots, 0\right) \in \mathrm{R}^{N \times N}$ such that the following conditions are satisfied:

1.

$\Omega=\left[\begin{array}{ccc}-D-P & \frac{1}{2} A+L & \frac{1}{2} B \\ \frac{1}{2} A^{T}+L & -I_{n} & 0 \\ \frac{1}{2} B^{T} & 0 & -I_{n}\end{array}\right]<0$

and

2.

$I_{N} \otimes P+c G \otimes \Gamma-c K \otimes \Gamma<0$.

Proof. First, denote

$e_{t}=e(t+\theta),-\tau \leq \theta \leq 0$.

Consider the following Lyapunov functional candidate:

$V\left(e_{t}\right)=\frac{1}{2} \sum_{i=1}^{N} e_{i}^{T}(t) e_{i}(t)+\sum_{i=1}^{N} \int_{t-\tau}^{t} g^{T}\left(e_{i}(\theta)\right) g\left(e_{i}(\theta)\right) d \theta$,

where $g\left(e_{i}(\cdot)\right)=f\left(x_{i}(\cdot)\right)-f(s(\cdot)) \quad(i=1,2, \cdots, N)$.

The derivative of $V(t)$ along the trajectories of (7) can be obtained as follows,

$\dot{V}\left(e_{t}\right)=\sum_{i=1}^{N} e_{i}^{T}(t) \dot{e}_{i}(t)+\sum_{i=1}^{N}\left[f\left(x_{i}(t)\right)-f(s(t))\right]^{T}\left[f\left(x_{i}(t)\right)-f(s(t))\right]$

$-\sum_{i=1}^{N}\left[f\left(x_{i}(t-\tau)\right)-f(s(t-\tau))\right]^{T} \times\left[f\left(x_{i}(t-\tau)\right)-f(s(t-\tau))\right]$
$=\sum_{i=1}^{N} e_{i}^{T}(t)\left\{-D e_{i}(t)+A\left[f\left(x_{i}(t)\right)-f(s(t))\right]+B\left[f\left(x_{i}(t-\tau)\right)-f(s(t-\tau))\right]\right.$

$\left.+c \sum_{j=1}^{N} G_{i j} \Gamma e_{j}(t)\right\}-c \sum_{i=1}^{l} k_{i} e_{i}^{T}(t) \Gamma e_{i}(t)$

$+\sum_{i=1}^{N}\left[f\left(x_{i}(t)\right)-f(s(t))\right]^{T}\left[f\left(x_{i}(t)\right)-f(s(t))\right]$

$-\sum_{i=1}^{N}\left[f\left(x_{i}(t-\tau)\right)-f(s(t-\tau))\right]^{T} \times\left[f\left(x_{i}(t-\tau)\right)-f(s(t-\tau))\right]$

From the assumption $(H)$, one has

$\left[f\left(x_{i}(t)\right)-f(s(t))\right]^{T}\left[f\left(x_{i}(t)\right)-f(s(t))\right]$

$\leq\left[x_{i}(t)-s(t)\right]^{T} L\left[f\left(x_{i}(t)\right)-f(s(t))\right]$

Combing (12)and (11), one obtains

$$
\begin{aligned}
& \dot{V}\left(e_{t}\right) \leq \sum_{i=1}^{N}\left\{e_{i}^{T}(t)(-D-P) e_{i}(t)+e_{i}^{T}(t)(A+2 L)\left[f\left(x_{i}(t)\right)-f(s(t))\right]\right. \\
& +e_{i}^{T}(t) B\left[f\left(x_{i}(t-\tau)\right)-f(s(t-\tau))\right]+\sum_{i=1}^{N}\left[f\left(x_{i}(t)\right)-f(s(t))\right]^{T}\left(-2 I_{n}+I_{n}\right) \\
& \times\left[f\left(x_{i}(t)\right)-f(s(t))\right]-\sum_{i=1}^{N}\left[f\left(x_{i}(t-\tau)\right)-f(s(t-\tau))\right]^{T} \\
& \left.\times\left[f\left(x_{i}(t-\tau)\right)-f(s(t-\tau))\right]\right\} \\
& +e^{T}(t)\left(I_{N} \otimes P+c G \otimes \Gamma-c K \otimes \Gamma\right) e(t) \\
& =\sum_{i=1}^{N} \eta_{i}^{T}(t) \Omega \eta_{i}(t)+e^{T}(t)\left(I_{N} \otimes P+c G \otimes \Gamma-c K \otimes \Gamma\right) e(t) \\
& \quad<0, \quad \begin{array}{c}
e_{i}(t) \\
\text { where } \eta_{i}(t)=\left[\begin{array}{c}
\left(f\left(x_{i}(t)\right)-f(s(t))\right) \\
\left(f\left(x_{i}(t-\tau)\right)-f(s(t-\tau))\right)
\end{array}\right],
\end{array} \\
& \text { and } e(t)=\left[e_{1}^{T}(t), e_{2}^{T}(t), \cdots, e_{N}^{T}(t)\right]^{T} .
\end{aligned}
$$

From (13), it is easy to see that the undirected network (5)globally synchronize under the given linear feedback pinning controllers. The proof is completed.

Remark 1. The condition (i) depends on the nonlinear dynamics of each single node. The condition (ii) shows that the key factors influencing the network synchronizability are the network inner linking matrix $(\Gamma)$, the network toplogical matrix $(G)$ and the pinning controller gains matrix $(K)$, in addition to the matrix $(P)$.

It is noted that the dimension of the matrix condition in (9) is $n N \times n N$, which can be further reduced as shown in the following corollary .

Corollary 1. Suppose that Assumption $(H)$ and condition (8) hold, and $\Gamma>0$. The controlled undirected network (5) globally synchronize to (4) if the following condition is satisfied:

$\Delta=\theta I_{N}+c(G-K)<0$

where ${ }^{\theta=\left\|P \Gamma^{-1}\right\|}$, the matrix $P$ is determined in Theorem 1, and $P \Gamma=\Gamma P$.

Proof. First, from Lemma 5 and $P \Gamma=\Gamma P$, one has 


$$
I_{N} \otimes P+c G \otimes \Gamma-c K \otimes \Gamma<\left(\theta I_{N}+c G-c K\right) \otimes \Gamma
$$

If (14) holds, i.e., $\theta I_{N}+c(G-K)<0$ and $\Gamma>0$, then one can easily get $\left(\theta I_{N}+c G-c K\right) \otimes \Gamma<0$, which implies (9).

Thus, conditions in Theorem 1 are satisfied, and the proof is thus completed.

Remark 2. The condition (14) is much simpler, and the dimension of matrix $\Delta$ is $N \times N$.

Next, some criteria for choosing an appropriate coupling strength are established.

Corollary 2. Suppose that Assumption $(\mathrm{H})$ holds and $\Gamma$ is a positive definite matrix and $\theta=\left\|P \Gamma^{-1}\right\|$ with $P \Gamma=\Gamma P$. The controlled undirected network (5) globally synchronize to (4) if the following condition is satisfied:

$$
c>\frac{\theta}{\left|\lambda_{\max }(G-K)\right|}
$$

Proof. By Lemma 4, it is easy to see that $G-K^{*}$ is negative definite, where $K^{*}$ corresponds to only one controller, i.e. $l=1$. It is easy to see that $\lambda_{\max }(G-K) \leq \lambda_{\max }\left(G-K^{*}\right)<0$. From (16), $c(G-K)+\theta I_{N} \leq\left(c \lambda_{\max }(G-K)+\theta\right) I_{N}<0$, so (14) is satisfied. The proof is completed.

Remark 3. It is easy to see that the condition for the coupling strength given in (16) is conservative, usually much larger than the needed value. For example in [29], it requires a very large coupling strength in general, which may not be very practical. Clearly, it is desirable to make coupling strength as small as possible. In [25], the cost function $c \sum_{i=1}^{l} k_{i}$ is introduced to describe the efficiency of the pinning controller and the coupling strength. The smaller the cost function, the more efficient the strategy to achieve the same goal of control, and the easier to be implemented. In order to make the coupling strength smaller, here, the adaptive control method can be adopted to achieve the goal. The pinning controllers in (6), associated with the following adaptive coupling law, lead to

$$
\begin{aligned}
& \dot{x}_{i}(t)=-D x_{i}(t)+A f\left(x_{i}(t)\right)+B f\left(x_{i}(t-\tau)\right)+I(t)+c(t) \sum_{j=1}^{N} G_{i j} \Gamma x_{j}(t) \\
& -c(t) k_{i} \Gamma\left(x_{i}(t)-s(t)\right), i=1,2, \cdots, l \\
& \dot{x}_{i}(t)=-D x_{i}(t)+A f\left(x_{i}(t)\right)+B f\left(x_{i}(t-\tau)\right)+I(t) \\
& +c(t) \sum_{j=1}^{N} G_{i j} \Gamma x_{j}(t), i=l+1, \cdots, N \\
& \dot{c}(t)=\alpha \sum_{j=1}^{N}\left(x_{j}(t)-s(t)\right)^{T} \Gamma\left(x_{j}(t)-s(t)\right)
\end{aligned}
$$

where $\alpha$ is a small positive constant.

\subsection{Directed Network}

The structure of a directed network is more complex than that of an undirected network, since a directed path may exist through the network from vertex $a$ to vertex $b$, but it does not guarantee that the path exists from $b$ to $a$. As a result, the coupling matrix is asymmetric. The controlled complex dynamical directed network can be written as

$$
\begin{aligned}
& \dot{\bar{x}}_{i}(t)=-D \bar{x}_{i}(t)+A f\left(\bar{x}_{i}(t)\right)+B f\left(\bar{x}_{i}(t-\tau)\right)+I(t) \\
& +c \sum_{j=1}^{N} \bar{G}_{i j} \Gamma \bar{x}_{j}(t)+u_{i}, i=1,2, \cdots, N
\end{aligned}
$$

where $\bar{G}=\left(\bar{G}_{i j}\right)_{N \times N} \in A_{1}$, and

$$
u_{i}=-c \bar{k}_{i} \Gamma\left(\bar{x}_{i}(t)-s(t)\right) \text {, }
$$

$\bar{k}_{i}>0, i=1,2, \cdots, l ;$ and $\bar{k}_{i}=0, i=l+1, \cdots, N ;$ Let $\bar{e}_{i}(t)=\bar{x}_{i}(t)-s(t)$, subtracting (4) from (18) yields the following error directed network:

$$
\begin{aligned}
& \dot{\bar{e}}_{i}(t)=-D \bar{e}_{i}(t)+A f\left(\bar{x}_{i}(t)\right)-A f(s(t))+B f\left(\bar{x}_{i}(t-\tau)\right)-B f(s(t-\tau)) \\
& +c \sum_{j=1}^{N} G_{i j} \Gamma \bar{e}_{j}(t)-c \bar{k}_{i} \Gamma \bar{\Gamma}_{i}(t) \\
& i=1,2, \cdots, N
\end{aligned}
$$

Theorem 2. Suppose that Assumption $(H)$ holds and $G \in A_{1}$. The controlled directed network (18) globally synchronizes to (4) if there is a symmetric matrix $\bar{P} \in \mathrm{R}^{n \times n} \quad$ and a diagonal nonnegative matrix $\bar{K}=\operatorname{diag}\left(\bar{k}_{1}, \bar{k}_{2}, \cdots, \bar{k}_{l}, 0,0 \cdots, 0\right) \in \mathrm{R}^{N \times N}$ such that the following conditions are satisfied:

\section{1.}

$\bar{\Omega}=\left[\begin{array}{ccc}-D-\bar{P} & \frac{1}{2} A+L & \frac{1}{2} B \\ \frac{1}{2} A^{T}+L & -I_{n} & 0 \\ \frac{1}{2} B^{T} & 0 & -I_{n}\end{array}\right]<0$

and

2.

$E \otimes \bar{P}+\frac{1}{2} c\left(E G+G^{T} E\right) \otimes \Gamma-c E K \otimes \Gamma<0$

where $E=\operatorname{diag}\left(\xi_{1}, \xi_{2}, \cdots, \xi_{N}\right), \quad \sum_{i=1}^{N} \xi_{i}=1, \quad$ and its diagonal elements are the left eigenvector of $G$ corresponding to the eigenvalue.

Proof. First, denote

$\bar{e}_{t}=\bar{e}(t+\theta),-\tau \leq \theta \leq 0$.

Consider the following Lyapunov functional:

$$
\bar{V}\left(\bar{e}_{t}\right)=\frac{1}{2} \sum_{i=1}^{N} \xi_{i} \bar{e}_{i}^{T}(t) \bar{e}_{i}(t)+\sum_{i=1}^{N} \xi_{i} \int_{t-\tau}^{t} g^{T}\left(\bar{e}_{i}(\theta)\right) g\left(\bar{e}_{i}(\theta)\right) d \theta,
$$

where

$$
g\left(\bar{e}_{i}(\cdot)\right)=f\left(\bar{x}_{i}(\cdot)\right)-f(s(\cdot)) .
$$

The derivative of $\bar{V}\left(\bar{e}_{t}\right)$ along the trajectories of (20) can be described as

$$
\begin{aligned}
& \dot{\bar{V}}\left(\bar{e}_{t}\right)=\frac{1}{2} \sum_{i=1}^{N} \xi_{i} \dot{\bar{e}}_{i}^{T}(t) \bar{e}_{i}(t)+\frac{1}{2} \sum_{i=1}^{N} \xi_{i} \bar{e}_{i}^{T}(t) \dot{\bar{e}}_{i}(t)+\sum_{i=1}^{N}\left[g^{T}\left(\bar{e}_{i}(t)\right) g\left(\bar{e}_{i}(t)\right)\right. \\
& \left.-g^{T}\left(\bar{e}_{i}(t-\tau)\right) g\left(\bar{e}_{i}(t-\tau)\right)\right]
\end{aligned}
$$


$=\frac{1}{2} \sum_{i=1}^{N} \xi_{i}\left\{\bar{e}_{i}^{T}(t)(-D) \bar{e}_{i}(t)+\bar{e}_{i}^{T}(t) A g\left(\bar{e}_{i}(t)\right)+g^{T}\left(\bar{e}_{i}(t)\right) A^{T} \bar{e}_{i}(t)\right.$

$+\bar{e}_{i}^{T}(t) B g\left(\bar{e}_{i}(t-\tau)\right)+g^{T}\left(\bar{e}_{i}(t-\tau)\right) B^{T} \bar{e}_{i}(t)$

$\left.+c \bar{e}_{i}^{T}(t) \sum_{j=1}^{N} G_{i j} \Gamma \bar{e}_{j}(t)+c \sum_{j=1}^{N} \bar{e}_{j}^{T}(t) \Gamma^{T} G_{i j} \bar{e}_{i}(t)\right\}$

$-\sum_{i=1}^{l} \xi_{i} \bar{e}_{i}^{T}(t) c k_{i} \Gamma \bar{e}_{i}(t)+\sum_{i=1}^{N}\left[g^{T}\left(\bar{e}_{i}(t)\right) g\left(\bar{e}_{i}(t)\right)-g^{T}\left(\bar{e}_{i}(t-\tau)\right) g\left(\bar{e}_{i}(t-\tau)\right)\right]$

Consider the following fact,

$\left[f\left(\bar{x}_{i}(t)\right)-f(s(t))\right]^{T}\left[f\left(\bar{x}_{i}(t)\right)-f(s(t))\right] \leq\left[\bar{x}_{i}(t)-s(t)\right]^{T} L\left[f\left(\bar{x}_{i}(t)\right)-f(s(t))\right]$.

Combing (24)and (25), adding and subtracting $\sum_{i=1}^{N} \xi_{i} \bar{e}_{i}(t)^{T} \bar{P} \bar{e}_{i}(t)$, we have

$\dot{\bar{V}}\left(\bar{e}_{t}\right) \leq \sum_{i=1}^{n} \xi_{i} \bar{\eta}_{i}^{T}(t) \bar{\Omega} \bar{\eta}_{i}(t)+\bar{e}^{T}(t)\left[E \otimes \bar{P}+\frac{1}{2} c\left(E G+G^{T} E\right) \otimes \Gamma\right.$

$-c E \bar{K} \otimes \Gamma] \bar{e}(t)$

$<0$,

where $\quad \bar{\eta}_{i}(t)=\left[\bar{e}_{i}^{T}(t),\left(f\left(\bar{x}_{i}(t)\right)-f(s(t))\right)^{T},\left(f\left(\bar{x}_{i}(t-\tau)\right)-f(s(t-\tau))\right)^{T}\right]^{T}$, and $\bar{e}(t)=\left[\bar{e}_{1}^{T}(t), \bar{e}_{2}^{T}(t), \cdots, \bar{e}_{N}^{T}(t)\right]^{T}$.

From the conditions (21) and (22), one knows that the network (18)synchronize to (4).

Similar to Corollary 1, a simpler condition for the directed networks can be obtained as follows.

Corollary 3. Suppose that Assumption $(H)$ and condition (21) hold, $\Gamma>0$. The controlled directed network (18) globally synchronizes to (4) if the following condition is satisfied:

$\bar{\Delta}=\bar{\theta} E+\frac{1}{2} c\left(E G+G^{T} E\right)-c E \bar{K}<0$

where $\bar{\theta}=\left\|\bar{P} \Gamma^{-1}\right\|$, the matrix $\bar{P}$ is determined in Theorem 2 and $\bar{P} \Gamma=\Gamma \bar{P}$.

\subsection{Pinning Scheme}

In this subsection, a unified pinning scheme is designed for undirected and directed networks when the network structure and coupling strength are fixed.

Theorem 3. Suppose that Assumption $(H)$ holds. If the control gains $k_{i}\left(\bar{k}_{i}\right)$ are chosen to be sufficiently large,

(1) The condition $\Delta<0$ in (14) is equivalent to $\tilde{\Delta}<0$;

(2) The condition $\bar{\Delta}<0$ in (27) is equivalent to $\tilde{\bar{\Delta}}<0$;

where $\tilde{\Delta}$ and $\tilde{\bar{\Delta}}$ are obtained by_removing the $1,2, \cdots, l$ row-column pairs of matrices $\Delta$ and $\bar{\Delta}$ respectively.

Proof. Let $\Delta=\theta I_{N}+c(G-K)=\left(\begin{array}{cc}Q-c \tilde{K} & R \\ R^{T} & \tilde{\Delta}\end{array}\right)$,

where $\left.\tilde{K}=\operatorname{diag}\left(k_{1}, k_{2}, \cdots, k_{l}\right)\right)$.

Obviously, if $\Delta<0$ then $\tilde{\Delta}<0$. So, we only need to prove that if $\tilde{\Delta}<0$ then $\Delta<0$. Since the control gains $k_{i}$ can be chosen to be sufficiently large, one can select $c \tilde{K}>Q-R \tilde{\Delta}^{-1} R^{T}$. From Schur complement, we have $\Delta<0$.

Let $\bar{\Delta}=\bar{\theta} E+\frac{1}{2}\left(E G+G^{T} E\right)-c E K=\left(\begin{array}{cc}\bar{Q}-c E \tilde{\bar{K}} & \bar{R} \\ \bar{R}^{T} & \tilde{\bar{\Delta}}\end{array}\right), \quad$ where $\tilde{\bar{K}}=\operatorname{diag}\left(\bar{k}_{1}, \bar{k}_{2}, \cdots, \bar{k}_{l}\right)$. The equivalence of $\bar{\Delta}<0$ and $\tilde{\bar{\Delta}}<0$ can be similarly proved. The proof is completed

It follows from the definitions of $\tilde{\Delta}$ and $\widetilde{\bar{\Delta}}$ that $\tilde{\Delta}=\theta I_{N-l}+c \tilde{G}, \tilde{\bar{\Delta}}=\bar{\theta} \tilde{E}+c \tilde{\bar{G}}$,

where $\tilde{G}_{i j}=G_{l+i, l+j}, \quad \tilde{\bar{G}}_{i j}=\left[\frac{1}{2}\left(E G+G^{T} E\right)\right]_{l+i, l+j}$ for $i, j=1,2, \cdots, N-l$, and $\tilde{E}=\operatorname{diag}\left(\xi_{l+1}, \xi_{l+2}, \cdots, \xi_{N}\right)$. Then one has the following corollary.

Corollary 4. Suppose that Assumption $(H)$ holds and $\Gamma$ is a diagonal positive definite matrix.

(i)The controlled undirected network (5) globally synchronizes to (4) if $\tilde{K}>\frac{\lambda_{\max }\left(Q-R \tilde{\Delta}^{-1} R^{T}\right)}{c} I_{l} \quad$ and $c \lambda_{\max }(\tilde{G})+\theta<0 ;$

(ii)The controlled directed network (18) globally synchronizes to (4) if $\quad \tilde{\bar{K}}>\frac{\lambda_{\max }\left(\bar{Q}-\bar{R}_{\overline{\bar{\Delta}}}^{-1} \bar{R}^{T}\right)}{c\left(\min _{1 \leq i s} \xi_{i}\right)} I_{l} \quad$ and $c \lambda_{\max }(\tilde{\bar{G}})+\bar{\theta}\left(\max _{l+1 \leq i \leq N} \xi_{i}\right)<0$.

Remark 4. The condition in Theorem 3 is very simple. It also provides some guidance to choose the control gains and therefore is very useful. The condition in Corollary 4 provides a criterion for ensuring the network synchronization with the network structure, coupling strength and pinning scheme in the form of (6) or (19).

Remark 5. In the above discussion, the synchronization is considered for the undirected network and the directed network via pinning control. In fact, the undirected network is the special case of the directed one, i.e., the matrix $E$ for the undirected network is $\operatorname{diag}\left(\frac{1}{N}, \frac{1}{N}, \cdots, \frac{1}{N}\right) \in R^{N \times N}$.

\section{NUMERICAL SIMULATIONS}

Consider the following 2-dimensional delayed neural networks

$\frac{d x(t)}{d t}=-D x(t)+A f(x(t))+B f(x(t-\tau))$,

where $x(t)=\left[x_{1}(t), x_{2}(t)\right]^{T}$ is the state vector of the network, $\boldsymbol{\tau}=1, f(x(t))=\left[\tanh \left(x_{1}\right), \tanh \left(x_{2}\right)\right]^{T}$, and

$D=\left[\begin{array}{ll}1 & 0 \\ 0 & 1\end{array}\right], \quad A=\left[\begin{array}{cc}2 & -0.1 \\ -5 & 3\end{array}\right], \quad B=\left[\begin{array}{ll}-1.5 & -0.1 \\ -0.2 & -2.5\end{array}\right]$,

$x_{1}(t) \equiv-0.4, x_{2}(t) \equiv 2, t \in[-1,0]$. The orbits of chaotic trajectory of (28) is illustrated in Fig. (1).

Now consider a scale-free network consisting of one hundred linearly coupled models, where $N=100$, $m_{0}=m=3$, the coupling strength $c=10$ and $\Gamma=I_{2}$. 


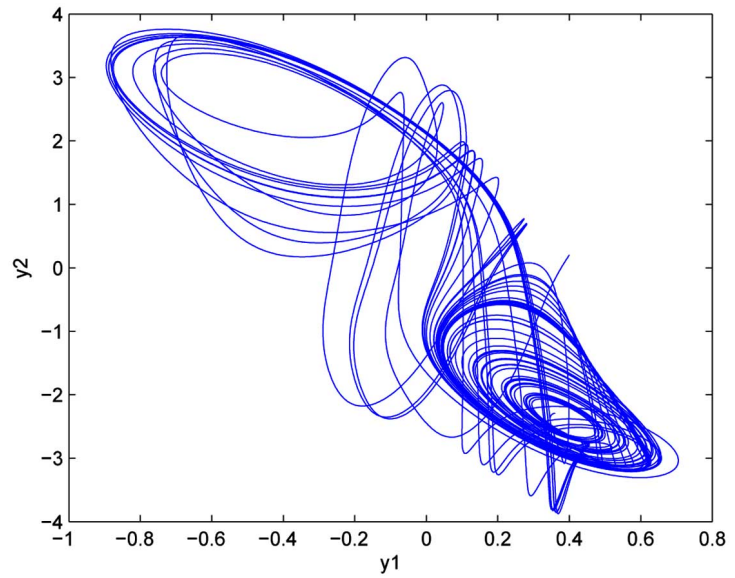

Fig. (1). The orbit of chaotic trajectory of (28).
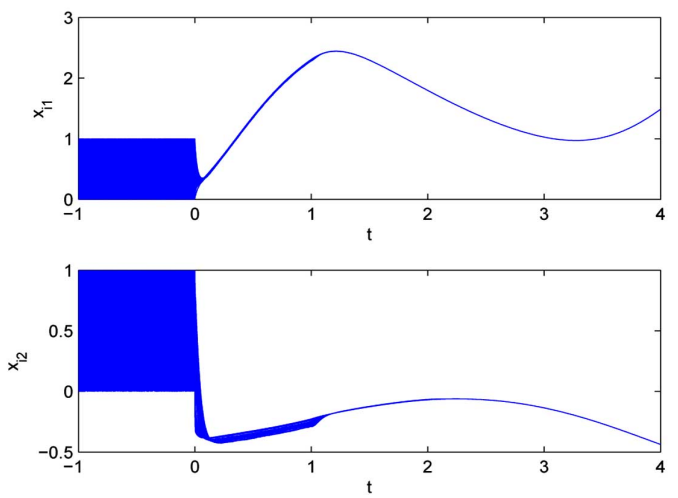

Fig. (2). The state of the scale-free network.
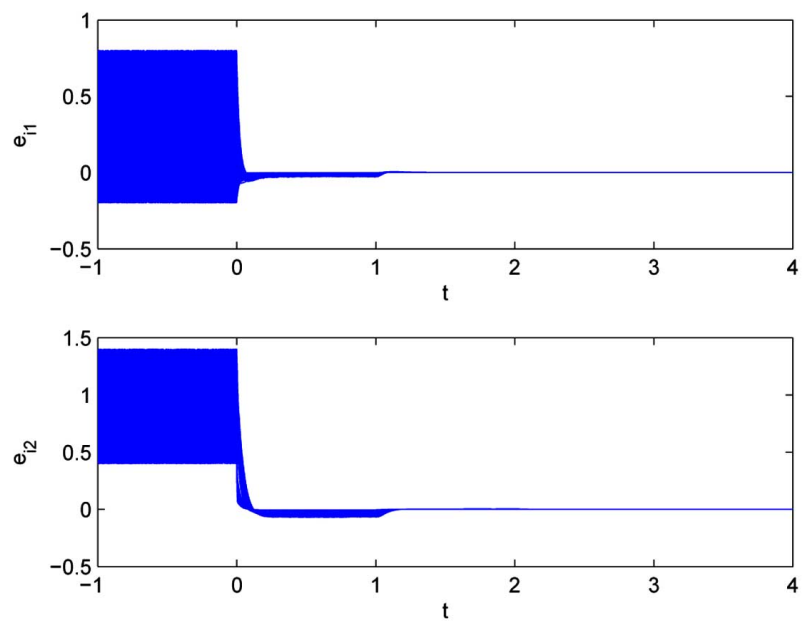

Fig. (3). Error states ei(t) in a scale -free network of (28).

Let twenty nodes are selected for pinning control and gains $k_{i}=40,(i=1,2, \cdots 20)$. The fact $\theta I+c(G-K)<0$ is easily verified, where $\theta=\left\|P \Gamma^{-1}\right\|=13.9674$ and the matrix $P=\left[\begin{array}{cc}23.7709 & 0 \\ 0 & 27.9348\end{array}\right]$ can be obtained from solving the

LMI (8). Therefore, the coupled network with the pinning controllers is globally synchronized. The closed-loop response and the synchronization state response are shown in Fig. (2) and the synchronization error is illustrated in Fig. (3).

\section{CONCLUSIONS}

In this paper, synchronization of undirected and directed networks has been investigated via pinning control. Some criteria for ensuring coupled networks synchronization have been derived, and some analytical techniques have been proposed to obtain appropriate coupling strength and control gain matrix for achieving network synchronization. Furthermore, the effective pinning schemes has been designed for networks with fixed structure and coupling strength. Finally, an numerical example has also been given to illustrate the theoretical analysis.

\section{APPENDIX}

Barabjäasi and Albert (BA) scale-free model. A significant discovery in the field of complex networks is the observation that a number of large-scale complex networks, including the Internet,WWW and metabolic networks, are scale free and their connectivity distributions have a powerlaw form. To explain the origin of power-law degree distribution, BA proposed another network model [2], the algorithm of which is given as follows:

(i) Growth. Start with a small number $\left(m_{0}\right)$ of nodes; at every time step, a new node is introduced and is connected to $m \leq m_{0}$ already-existing nodes. (In this paper, we use $\mathrm{m}=\mathrm{m}_{0}$ - 3for convenience).

(ii) Preferential attachment. The probability $\Pi_{i}$ that a new node will be connected to node $\mathrm{i}$ (one of the $\mathrm{m}$ alreadyexisting nodes) depends on the degree $k_{i}$ of node $i$, in such a way that $\Pi_{i}=k_{i} / \Sigma_{j} k_{j}$.

\section{CONFLICT OF INTRREST}

None declared.

\section{ACKNOWLEDGEMENT}

This work was jointly supported by a grant from RGC of Hong Kong (CityU:113708), the National Natural Science Foundation of China under Grants No. 11072059 and No. 61004043, and the Specialized Research Fund for the Doctoral Program of Higher Education under Grants No. 20110092110017 and No. 2009092120066.

\section{REFERENCES}

[1] D. Watts, S. Strogatz, "Collective dynamics of small-world networks", Nature., vol. 393, pp. 440-442, 1998.

[2] A. Barabasi, R. Albert, "Emergence of scaling in random networks", Science., vol. 286, pp. 509-512, 1999.

[3] P. Erdos, A. Renyi, "On random graphs", Pub. Math., vol. 6, pp. 290-297, 1959.

[4] P. Erdos, A. Renyi, "On the evolution of random graphs", Pub. Math. Inst. Hungariam. Acad. Sci., vol. 5, pp. 17-61, 1960.

[5] R. Olfati-Saber, R.Murray, "Consensus problems in networks of agents with switching topology and time-delays", IEEE Trans. Automat. Contr., vol. 49, no. 9, pp. 1520-1533, 2004.

[6] R.A. Horn, C. Johnson, Matrix Analysis, Cambridge University Press: Cambridge, England, 1987.

[7] W. Li, "Stability analysis of swarms with general topology", IEEE Trans. Syst. Man. Cybern. B: Cybernetics, vol. 38, no. 4, pp. 10841097, 2008.

[8] C. Wu, L. Chua, "Synchronization in an array of linearly coupled dynamical systems", IEEE Trans. Circuits Syst. I., vol. 42, no. 8, pp. $430-447,1995$. 
[9] C. Wu, "Synchronization in coupled arrays of chaotic oscillators with nonreciprocal coupling", IEEE Trans. Circuits Syst. I., vol. 50, no. 2, pp. 294-297, 2003.

[10] C. Wu, "Synchronization in arrays of coupled nonlinear systems with delay and nonreciprocal time-varying coupling”, IEEE Trans. Circuits Syst.-II., vol. 52, no. 5, pp. 282-286, 2005.

[11] D. Terman, E. Lee, "Partial Synchronization in a network of neural oscillators”, SIAM J. Appl. Math., vol. 57, no. 1 pp. 252-293, 1997.

[12] E. Izhikevich, F. Hoppensteadt, "Slowly coupled oscillators: phase dynamics and synchronization", SIAM J. Appl. Math., vol. 63, no. 6, pp. 1935-1953, 2003.

[13] J. Lü, G. Chen, "A time-varying complex dynamical model and its controlled synchronization criteria”, IEEE Trans. Automat. Contr., vol. 50 , no. 6 , pp. 841-846, 2005.

[14] W. Lu, T. Chen, "New approach to synchronization analysis of linearly coupled ordinary differential systems", Physica D., vol. 213, pp. 214-230, 2006.

[15] C. Li, G. Chen, "Synchronization in general complex dynamical networks with coupling delays", Physica A., vol. 343, pp. 263-278, 2004.

[16] W. Lu, T. Chen, "Synchronization of coupled connected neural networks with delays", IEEE Trans. Circuits Syst.-I., vol. 51, no. 12, pp. 2491-2503, 2004.

[17] G. Chen, J. Zhou, Z. Liu, "Global synchronization of coupled delayed neural networks and applications to chaos CNN models", Int. J. Bifurcat. Chaos., vol. 14, no. 7, pp. 2229-2240, 2004.

[18] W. Wang, J. Cao, "Synchronization in an array of linearly coupled networks with time-varying delay", Physica A., vol. 366, pp. 197211, 2006.

[19] J. Cao, G. Chen, P. Li, "Global synchronization in an array of delayed neural networks with hybrid coupling”, IEEE Trans. Syst. Man, Cybern. B: Cybernetics, vol. 38, no. 2, pp. 488-498, 2008.

[20] W. Yu, J. Cao, J. Lv, "Global synchronization of linearly hybrid coupled networks with time-varying delay", SIAM J. Appl. Dyn. Sys. vol. 7, no. 1, pp. 108-133, 2008.
[21] W. Yu, J. Cao, G. Chen, J. Lu, J. Han, W. Wei, "Local synchronization of a complex network model", IEEE Trans. Syst. Man, Cybern. B: Cybernetics, vol. 39, no. 1, pp. 230-241, 2009.

[22] K. Yuan, "Robust synchronization in arrays of coupled networks with delay and mixed coupling", Neurocomputing., vol.72, no. 4-6, pp. 1026-1031, 2009.

[23] J. Cao, P. Li, W. Wang, "Global synchronization in arrays of delayed neural networks with constant and delayed coupling", Phys. Lett. A., vol. 353, no. 4, pp. 318-325, 2006.

[24] R. Grigoriev, M. Cross, H. Schuster, "Pinning control of spatiotemporal chaos", Phys. Rev. Lett., vol. 79, no. 15, pp. 27952798, 1997.

[25] R. Li, G. Chen, Z. Duan, "Cost and effect of pinning control for network synchronization", Chinese Physics B., in press.

[26] W. Yu, G. Chen, J Lü, "On pinning synchronization of complex dynamical networks", Automatica., vol. 45, no. 2, pp. 429-435, 2009

[27] J. Lu, D.W.C. Ho, J.Cao, J. Kurths. "Exponential synchronization of linearly coupled neural networks with impulsive disturbances", IEEE Trans. Neural Netw., vol. 22, no. 2, pp. 329-335, 2011.

[28] J. Lu, D.W.C. Ho. "Stabilization of complex dynamical networks with noise disturbance under performance constraint", Nonlinear Anal., B: Real World Appl., vol. 12, pp.1974-1984, 2011.

[29] T. Chen, X. Liu, W. Lu, "Pinning complex networks by a single controller", IEEE Trans. Circuits Syst. I., vol. 54, no. 6, pp. 13171326, 2007.

[30] X. Wang, G. Chen, "Pinning control of scale-free dynamical networks", Physica A., vol. 310, pp. 521-531, 2002.

[31] X. Li, X. Wang, G. Chen, "Pinning a complex dynamical network to its equilibrium”, IEEE Trans. Circuits Syst. I., vol. 51, no. 10, pp. 2074-2087, 2004.

[32] J. Zhou, J. Lu, J. Lü, "Pinning adaptive synchronization of a general complex dynamical network", Automatica., vol. 44, no. 4, pp. 996-1003, 2008.

Received: December 14, 2011

Revised: February 11, 2012

Accepted: February 21, 2012

(C) Cao et al.; Licensee Bentham Open.

This is an open access article licensed under the terms of the Creative Commons Attribution Non-Commercial License (http://creativecommons.org/licenses/by-nc/3.0/) which permits unrestricted, non-commercial use, distribution and reproduction in any medium, provided the work is properly cited. 